\title{
Dispersion coefficients for alkali-metal dimers
}

\section{Citation}

Marinescu, M., H. R. Sadeghpour, and A. Dalgarno. 1994. “Dispersion Coefficients for AlkaliMetal Dimers." Physical Review A 49 (2): 982-88. https://doi.org/10.1103/physreva.49.982.

\section{Permanent link}

http://nrs.harvard.edu/urn-3:HUL.InstRepos:41417401

\section{Terms of Use}

This article was downloaded from Harvard University's DASH repository, and is made available under the terms and conditions applicable to Other Posted Material, as set forth at http:// nrs.harvard.edu/urn-3:HUL.InstRepos:dash.current.terms-of-use\#LAA

\section{Share Your Story}

The Harvard community has made this article openly available.

Please share how this access benefits you. Submit a story.

Accessibility 


\title{
Dispersion coefficients for alkali-metal dimers
}

\author{
M. Marinescu, H. R. Sadeghpour, and A. Dalgarno \\ Harvard-Smithsonian Center for Astrophysics, 60 Garden Street, Cambridge, Massachusetts 02138
}

(Received 23 August 1993)

\begin{abstract}
Knowledge of the long-range interaction between atoms and molecules is of fundamental importance for low-energy and low-temperature collisions. The electronic interaction between the charge distributions of two ground-state alkali-metal atoms can be expanded in inverse powers of $R$, the internuclear distance. The coefficients $C_{6}, C_{8}$, and $C_{10}$ of, respectively, the $R^{-6}, R^{-8}$, and $R^{-10}$ terms are calculated by integrating the products of the dynamic electric multipole polarizabilities of the individual atoms at imaginary frequencies, which are in turn obtained by solving two coupled inhomogeneous differential equations. Precise one-electron model potentials are developed to represent the motion of the valence electron in the field of the closed alkali-metal positive-ion core. The numerical results for the static multipole polarizabilities for the alkali-metal atoms and the coefficients $C_{6}, C_{8}$, and $C_{10}$ for homonuclear and heteronuclear alkali-metal diatoms are compared with other calculations.
\end{abstract}

PACS number(s): 34.20. $-\mathrm{b}, 34.40 .+\mathrm{n}, 82.20 . \mathrm{Kh}$

\section{INTRODUCTION}

Advances in laser cooling and trapping technology have rekindled interest in the knowledge of the longrange forces between atoms and molecules. Confinement of alkali-metal atoms at sub-Kelvin temperatures depends on their determination of the asymptotic long-range properties [1-3]. At large separations, the atomic charge distributions hardly overlap and the internuclear potential $V_{a b}(R)$ between two atoms $a$ and $b$ can be represented as a sum of electronic potentials. This interaction between the charge distributions of two atoms or molecules can be expanded in a power of $R^{-1}$, where $R$ is the separation distance between the charge centers. Each term in this series corresponds to a particular multipole moment of the charge-charge interaction [4-9].

In this work, we concentrate on the multipole moments which arise in the second order of perturbation expansion, and determine the coefficients for the dipoledipole interaction $C_{6}$, the dipole-quadrupole interaction $C_{8}$, and the dipole-octupole and quadrupole-quadrupole interaction $C_{10}$ in the expansion

$$
V_{a b}(R)=-\frac{C_{6}}{R^{6}}-\frac{C_{8}}{R^{8}}-\frac{C_{10}}{R^{10}}-\cdots .
$$

The calculation of the long-range interactions between atoms can be reduced to the evaluation of electric dynamic multipole polarizabilities at imaginary frequencies [10-12]. Many theoretical studies have been reported using a variety of methods on the evaluation of the multipole polarizabilities of the alkali-metal atoms [13-26], and it has been demonstrated that model potential methods are capable of achieving high accuracy [18].

In this paper we present a method for computing dynamic multipole polarizabilities at imaginary frequencies that is exact given the assumption of a model potential for the motion of a valence electron in the presence of a frozen core. The infinite second-order sums are transformed into integrals over the solutions of two coupled inhomogeneous differential equations. Section II contains a brief derivation of the expression for the multipole coefficients and a detailed discussion of the numerical procedure for solving the coupled inhomogeneous equations. The numerical results including values for the electric multipole polarizabilities, and the dispersion coefficients for the homonuclear and heteronuclear cases, are presented in Sec. III, followed by a discussion. A detailed comparison with other theoretical values is included.

\section{FORMULATION OF THE PROBLEM}

\section{A. Theory}

The coefficients of expansion in Eq. (1) are conveniently represented in the compact form [12]

$$
\begin{aligned}
C_{2 n+2}=-\frac{1}{2 \pi} \sum_{k=1}^{n-1}\left[\sum_{j=-\min (k, k-n)}^{j=\min (k, k-n)}\left[\begin{array}{c}
n \\
k+j
\end{array}\right]\left[\begin{array}{c}
n \\
k-j
\end{array}\right]\right] \\
\times \int_{0}^{\infty} \alpha_{(n-k)}(i \omega) \alpha_{k}(i \omega) d \omega \text { for } n \geq 2
\end{aligned}
$$

where $\alpha_{l}(i \omega)$ are the dynamic $2^{l}$ polarizabilities at imaginary frequencies:

$\alpha_{l}(i \omega)=\frac{4 \pi}{2 l+1}\left[\sum_{s} \frac{\left|\left\langle 0\left|r_{0}^{(l)}\right| s\right\rangle\right|^{2}}{E_{s}-E_{0}+\omega}+\sum_{s} \frac{\left|\left\langle 0\left|r_{0}^{(l)}\right| s\right\rangle\right|^{2}}{E_{s}-E_{0}-\omega}\right]$.

The initial state is represented by the eigenstate $|0\rangle$ and eigenenergy $E_{0}$. The sum in Eq. (3) is over all possible discrete and continuum states. $r_{0}^{(l)}$ represents a tensor of rank $l$ and zero magnetic quantum number. Its alternative definition is $r_{0}^{(l)}=r^{l} Y_{l 0}(\hat{\mathrm{r}})$, where $Y_{l m}$ are the usual spherical harmonics.

The sums in Eq. (3) can be evaluated exactly by trans- 
forming into a differential equation form as

$$
\alpha_{l}(i \omega)=\frac{8 \pi}{2 l+1} \operatorname{Re}\left\langle 0\left|r_{0}^{(l)} G\left(E_{0}-i \omega\right) r_{0}^{(l)}\right| 0\right\rangle,
$$

where $G\left(E_{0}-i \omega\right)$ is the Green's function evaluated at complex energies, and $R e$ represents the real part of the matrix element. Upon carrying out the angular integration of the matrix element we obtain

$\alpha_{l}(i \omega)=\frac{2}{2 l+1} \operatorname{Re} \int_{0}^{\infty} R_{0}(r) \mathcal{F}_{l}\left(E_{0}-i \omega ; r\right) r^{l+2} d r$,

where

$\mathcal{F}_{l}\left(E_{0}-i \omega ; r\right)=\frac{1}{r} \int_{0}^{\infty} g_{l}\left(E_{0}-i \omega ; r, r^{\prime}\right) R_{0}\left(r^{\prime}\right) r^{\prime l+1} d r^{\prime}$

and $R_{0}$ is the radial wave function for the ground state and $g_{l}$ is the radial Green's function for the multipole order of $l$. Equation (6) can be written as an inhomogeneous differential equation [27]

$$
\begin{aligned}
& {\left[\frac{1}{r^{2}} \frac{d}{d r}\left[r^{2} \frac{d}{d r}\right]-\frac{l(l+1)}{r^{2}}\right.} \\
& \left.\quad+2\left[E_{0}-i \omega-V_{l}(r)\right]\right] \mathscr{F}_{l}\left(E_{0}-i \omega ; r\right)=2 r^{l} R_{0}(r)
\end{aligned}
$$

where the central field potential $V_{l}(r)$ is in general an $l$ dependent function of the radial coordinate. The boundary conditions on the solution of Eq. (7) can be extracted from the integral expression for $\mathscr{F}_{l}$ in Eq. (6). In the small-r region $\mathscr{F}_{l} \rightarrow$ const and for the large- $r$ region $F_{1} \rightarrow 0$. Because the energy in Eq. (7) is complex, solutions of Eq. (7) take on the complex form

$$
\mathcal{F}_{l}=\frac{1}{r}\left(\chi_{l}^{(R)}-i \chi_{l}^{(I)}\right)
$$

Substituting Eq. (8) into Eq. (7), we find the following coupled system of second-order inhomogeneous differential equations:

$$
\begin{aligned}
& {\left[\frac{d^{2}}{d r^{2}}-\frac{l(l+1)}{r^{2}}+2\left[E_{0}-V_{l}(r)\right]\right] \chi_{l}^{(R)}(r)} \\
& -2 \omega \chi_{l}^{(I)}(r)=2 r^{l+1} R_{0}(r), \\
& {\left[\frac{d^{2}}{d r^{2}}-\frac{l(l+1)}{r^{2}}+2\left[E_{0}-V_{l}(r)\right] \chi_{l}^{(I)}(r)+2 \omega \chi_{l}^{(R)}(r)=0\right. \text {. }}
\end{aligned}
$$

From Eq. (8), it can be seen that $\chi_{l}^{(R, I)}$ should satisfy the same boundary conditions as for $F_{l}$. Upon determining the solutions of Eqs. (9), the evaluation of the multipole polarizability reduces to a one-dimensional integral:

$$
\alpha_{l}(i \omega)=\frac{2}{2 l+1} \int_{0}^{\infty} R_{0}(r) \chi_{l}^{(R)}(r) r^{l+1} d r
$$

The multipole expansion coefficients are obtained from Eq. (2) by substituting for the polarizabilities from Eq. (10) and performing the integration over $\omega$. At $\omega=0$, the system of differential equations in (9) becomes decoupled and the imaginary part of $\mathcal{F}_{l}$ becomes identically zero as the second differential equation does not possess a solution for $l \neq 0$ at energy $E_{0}$.

\section{B. Numerical method for solving coupled inhomogeneous differential equations}

We opted to use the Numerov method to solve the set of coupled inhomogeneous equations

$$
\frac{d^{2}}{d r^{2}} \mathbf{y}=\mathbf{F y}+\mathbf{g},
$$

where $\mathbf{y}$ is the solution vector and $\mathbf{g}$ contains the inhomogeneities. For the problem at hand, $\mathbf{y}, \mathbf{F}$, and $\mathrm{g}$ have the following explicit expressions:

$$
\begin{aligned}
& \mathrm{y}(r) \equiv\left[\begin{array}{l}
\chi_{l}^{(R)}(r) \\
\chi_{l}^{(I)}(r)
\end{array}\right], \quad \mathrm{g}(r) \equiv\left[\begin{array}{cc}
2 r^{l+1} R_{0}(r) \\
0
\end{array}\right], \\
& \mathrm{F}(r) \equiv\left[\begin{array}{cc}
\frac{l(l+1)}{r^{2}}-2\left[E_{0}-V_{l}(r)\right] & 2 \omega \\
-2 \omega & \frac{l(l+1)}{r^{2}}-2\left[E_{0}-V_{l}(r)\right]
\end{array}\right] .
\end{aligned}
$$

In matrix notation, the Numerov recursion formula for the coupled inhomogeneous equations takes the form [28]

$$
\begin{aligned}
(1- & \left.\frac{h^{2}}{12} \mathbf{F}_{n+1}\right] \mathbf{y}_{n+1}-2\left[1+\frac{5 h^{2}}{12} \mathbf{F}_{n}\right] \mathbf{y}_{n} \\
& +\left[1-\frac{h^{2}}{12} \mathbf{F}_{n-1}\right] \mathbf{y}_{n-1}=\frac{h^{2}}{12}\left(\mathrm{~g}_{n+1}+10 \mathrm{~g}_{n}+\mathbf{g}_{n-1}\right),
\end{aligned}
$$

where $h$ is the step size and $\mathbf{F}_{m}, \mathbf{g}_{m}$, and $\mathbf{y}_{m}$ are the functional values of the matrix $\mathbf{F}$ and vectors $g$ and $\mathbf{y}$, evaluated at the $m$ th point on the radial scale. Equation (13) can be rewritten as a tridiagonal system of linear inhomogeneous equations where all the coefficients are matrices. Upon applying the boundary conditions on $y$, the solutions at the $k$ th radial point, $\mathbf{y}_{k}$, are obtained.

Due to the chaotic sensitivity of the inhomogeneous solutions to the initial conditions (i.e., near the origin), special care must be taken to ensure that the numerical 
solutions are stable. This task is made more difficult by the singular nature of the potentials arising in atomic physics. We therefore exercise care in choosing the grid scheme for numerical integration. The commonly used square root and arctangent mesh schemes, though quite useful for solving homogeneous equations, do not provide sufficient grid-point density near the origin for the solution of inhomogeneous equations. We chose the logarithmic scale $x=\ln r$ near the origin. As $r \rightarrow 0$, the range of integration will tend to increasing negative $x$ values such that even a modest step size of $10^{-2}$ in the negative $x$ direction will result in a very small step size on the $r$ scale.

Equations (11) are integrated along the $x$ axis in the range $x \in[-30,0.53]$ corresponding to $r \in\left[10^{-13}, 1.7\right]$ a.u., and then switched to the linear $r$ scale. The transformed equations in the $x$ coordinates are

$$
\frac{d^{2}}{d r^{2}} \tilde{y}=\widetilde{F} \mathbf{y}+\widetilde{g},
$$

where now

$$
\begin{aligned}
& \widetilde{\boldsymbol{y}}(x) \equiv\left[\begin{array}{l}
\widetilde{\chi}_{l}^{(R)}(x) \\
\widetilde{\chi}_{l}^{(I)}(x)
\end{array}\right], \quad \widetilde{g}(x) \equiv\left[\begin{array}{c}
2 e^{(l+5 / 2) x} R_{0}\left(e^{x}\right) \\
0
\end{array}\right] \\
& \widetilde{\mathbf{F}}(x) \equiv\left(\begin{array}{ccc}
l(l+1)+\frac{1}{4}-2 e^{2 x}\left[E_{0}-V\left(e^{x}\right)\right] & 2 e^{2 x} \omega \\
-2 e^{2 x} \omega & l(l+1)+\frac{1}{4}-2 e^{2 x}\left[E_{0}-V\left(e^{x}\right)\right]
\end{array}\right)
\end{aligned}
$$

such that the relation between $y(r)$ and $\mathscr{y}(x)$ is

$$
y(r)=\exp (x / 2) \tilde{y}(x) .
$$

The accuracy of the numerical scheme was tested for the following case. Let $V_{l}(r)=-1 / r$ in Eqs. (9). $E_{0}$ and $R_{0}(r)$ are then the ground-state energy and wave function for the hydrogen atom. Defining $u_{0}=r R_{0}$ and $u_{1}$ as the reduced wave functions for the $1 s$ and $2 p$ states of hydrogen, we premultiply Eqs. (9) once by $u_{0}$ and again by $u_{1}$ and integrate over $r$ to obtain the following four identities:

$$
\begin{aligned}
& \begin{array}{l}
\int_{0}^{\infty} u_{0}(r) \chi_{1}^{(R)}(r) \frac{d r}{r^{2}}+\omega \int_{0}^{\infty} u_{0}(r) \chi_{1}^{(I)}(r) d r \\
\quad+\int_{0}^{\infty} u_{0}^{2}(r) r d r=0,
\end{array} \\
& \int_{0}^{\infty} u_{0}(r) \chi_{1}^{(I)}(r) \frac{d r}{r^{2}}-\omega \int_{0}^{\infty} u_{0}(r) \chi_{1}^{(R)}(r) d r=0, \\
& \left(E_{1}-E_{0}\right) \int_{0}^{\infty} u_{1}(r) \chi_{1}^{(R)}(r) d r \\
& +\omega \int_{0}^{\infty} u_{1}(r) \chi_{1}^{(I)}(r) d r+\int_{0}^{\infty} u_{1}(r) u_{0}(r) r d r=0,
\end{aligned}
$$

$$
\left(E_{1}-E_{0}\right) \int_{0}^{\infty} u_{1}(r) \chi_{1}^{(I)}(r) d r-\omega \int_{0}^{\infty} u_{1}(r) \chi_{1}^{(R)}(r) d r=0
$$

Although $u_{0}$ and $u_{1}$ are known analytically, in order to test our numerical efficiency we continue to compute $E_{0}$, $E_{1}, u_{0}$, and $u_{1}$. We find that these identifies are satisfied to within one part in $10^{9}$. A more stringent test is however provided by the static dipole, quadrupole, and octupole polarizabilities arising in hydrogen-hydrogen interaction, for which analytic results are available. Our numerical values for the multipole polarizabilities of hydrogen $\alpha_{1}=4.500, \alpha_{2}=15.000$, and $\alpha_{3}=131.250$ agree with the analytical results of Chan and Dalgarno [29]. The corresponding values for the dispersion coefficients $C_{6}, C_{8}$, and $C_{10}$ in hydrogen are 6.499, 124.399, and 3285.833 , in agreement with Chan and Dalgarno who give $6.499,124.400$, and 3285.500 [29].

\section{Parametric model potential}

To describe the motion of the valence electron for the alkali-metal atoms we developed a model potential along the lines of Laughlin and Victor [30] and Greene and Aymar [31]. These potentials which are parametrically fitted to one-electron energy levels have been successful in predicting many observed phenomena such as the twoelectron resonances in the spectra of alkali-metal cations [31] and alkaline-earth atoms [32].

The form of this potential which can depend also on the orbital angular momentum of the valence electron, $l$, is

$$
V_{l}(r)=-\frac{Z_{l}(r)}{r}-\frac{\alpha_{c}}{2 r^{4}}\left[1-e^{-\left(r / r_{c}\right)^{6}}\right],
$$

where $\alpha_{c}$ is the static dipole polarizability of the positive-ion core while the radial charge $Z_{l}(r)$ is given by

$$
Z_{l}(r)=1+(z-1) e^{-a_{1} r}-r\left(a_{3}+a_{4} r\right) e^{-a_{2} r},
$$

where $z$ is the nuclear charge of the neutral atom and $r_{c}$ is the cutoff radius introduced to truncate the unphysical short-range contribution of the polarization potential near the origin. The potential in (18) is $l$ dependent, but this does not introduce any practical difficulties.

The five-parameter nonlinear fit of Eqs. (18), with $\alpha_{c}$ kept fixed, to the alkali-metal-atom Rydberg energies were carried out. The optimized parameters in (18) are listed in Table I. For each value of angular momentum $l$, 
TABLE I. Optimized parameters for the $l$-dependent model potential.

\begin{tabular}{|c|c|c|c|c|c|c|}
\hline$\alpha_{c}$ & & $\begin{array}{c}\mathbf{L i} \\
0.1923 \\
\end{array}$ & $\begin{array}{r}\mathrm{Na} \\
0.9448 \\
\end{array}$ & $\begin{array}{c}\mathbf{K} \\
5.3310\end{array}$ & $\begin{array}{c}\mathbf{R b} \\
9.0760\end{array}$ & $\begin{array}{c}\mathrm{Cs} \\
15.6440 \\
\end{array}$ \\
\hline \multirow[t]{5}{*}{$l=0$} & $a_{1}$ & 2.47718079 & 4.82223117 & 3.56079437 & 3.69628474 & 3.49546309 \\
\hline & $a_{2}$ & 1.84150932 & 2.45449865 & 1.83909642 & 1.64915255 & 1.47533800 \\
\hline & $a_{3}$ & -0.02169712 & -1.12255048 & -1.74701102 & -9.86069196 & -9.72143084 \\
\hline & $a_{4}$ & -0.11988362 & -1.42631393 & -1.03237313 & 0.19579987 & 0.02629242 \\
\hline & $r_{c}$ & 0.61340824 & 0.45489422 & 0.83167545 & 1.66242117 & 1.92046930 \\
\hline \multirow[t]{5}{*}{$l=1$} & $a_{1}$ & 3.45414648 & 5.08382502 & 3.65670429 & 4.44088978 & 4.69366096 \\
\hline & $a_{2}$ & 2.55151080 & 2.18226881 & 1.67520788 & 1.92828831 & 1.71398344 \\
\hline & $a_{3}$ & -0.21646561 & -1.19534623 & -2.07416615 & -16.79597770 & -24.65624280 \\
\hline & $a_{4}$ & -0.06990078 & -1.03142861 & -0.89030421 & -0.81633314 & -0.09543125 \\
\hline & $r_{c}$ & 0.61566441 & 0.45798739 & 0.85235381 & 1.50195124 & 2.13383095 \\
\hline \multirow[t]{5}{*}{$l=2$} & $a_{\mathrm{t}}$ & 2.51909839 & 3.53324124 & 4.12713694 & 3.78717363 & 4.32466196 \\
\hline & $a_{2}$ & 2.43712450 & 2.48697936 & 1.79837462 & 1.57027864 & 1.61365288 \\
\hline & $a_{3}$ & 0.32505524 & -0.75688448 & -1.69935174 & -11.65588970 & -6.70128850 \\
\hline & $a_{4}$ & 0.10602430 & -1.27852357 & -0.98913582 & 0.52942835 & -0.74095193 \\
\hline & $r_{c}$ & 2.34126273 & 0.71875312 & 0.83216907 & 4.86851938 & 0.93007296 \\
\hline \multirow[t]{5}{*}{$l \geq 3$} & $a_{1}$ & 2.51909839 & 1.11056646 & 1.42310446 & 2.39848933 & 3.01048361 \\
\hline & $a_{2}$ & 2.43712450 & 1.05458759 & 1.27861156 & 1.76810544 & 1.40000001 \\
\hline & $a_{3}$ & 0.32505524 & 1.73203428 & 4.77441476 & -12.07106780 & -3.20036138 \\
\hline & $a_{4}$ & 0.10602430 & -0.09265696 & -0.94829262 & 0.77256589 & 0.00034538 \\
\hline & $r_{s}$ & 2.34126273 & 28.6735059 & 6.50294371 & 4.79831327 & 1.99969677 \\
\hline
\end{tabular}

a minimum of five measured energies were used in the fitting procedure. The accuracy achieved in this calculation was one part in $10^{5}$.

\section{Core polarizability}

If the motion of the outer valence electron in the presence of the core is taken, in the spirit of BornOppenheimer approximation, to be adiabatic, then it can be shown [33] that the core perturbation due to the external electric field of the valence electron manifests itself in a correction to the multipole operator $\widetilde{Q}_{l}$ as

$$
\bar{Q}_{l} \rightarrow \widetilde{Q}_{l}\left(1-\frac{\widetilde{\alpha}_{c}^{(l)}}{r^{2 l+1}}\left[1-e^{-\left(r / r_{c}^{\prime}\right)^{2 l+1}}\right]\right)
$$

where $\widetilde{\alpha}_{c}^{(l)}$ is the core $2^{l}$ tensor polarizability and $r_{c}^{\prime}$ is a cutoff radius to be determined empirically. We have chosen it so that the corrected values of the neutral alkali-metal static dipole polarizabilities are in agreement with the experimental data [34]. The numerical values of $r_{c}^{\prime}$, are, respectively, $2.3542392,0.3798660,4.4395871$, 4.3397730 , and 4.9164157 for $\mathrm{Li}, \mathrm{Na}, \mathrm{K}, \mathrm{Rb}$, and $\mathrm{Cs}$, producing substantial corrections to the electric dipole moments.

\section{DISCUSSION OF RESULTS}

The dipole polarizabilities calculated with and without the core polarizability correction are listed in Table II. By construction, the corrected values are in agreement

TABLE II. Dipole polarizabilities $\alpha_{1}\left(a_{0}^{3}\right)$.

\begin{tabular}{|c|c|c|c|c|c|}
\hline Source & $\mathbf{L i}$ & $\mathrm{Na}$ & $\mathrm{K}$ & $\mathbf{R b}$ & Cs \\
\hline [13] & 170.0 & & & & \\
\hline$[18]$ & 164.3 & 162.6 & 298.0 & 333.0 & 416.5 \\
\hline$[21]$ & 163.7 & 162.4 & 287.1 & & \\
\hline$[22]$ & 165.8 & & & & \\
\hline$[24]$ & 164.1 & & & & \\
\hline$[25]$ & & & & 343.3 & 440.6 \\
\hline$[35]$ & & & 295.6 & 334.0 & 413.7 \\
\hline [38] & 165.0 & 151.0 & 292.0 & 328.0 & 405 \\
\hline experiment & $164.0 \pm 3.4$ & $159.2 \pm 3.4$ & $292.8 \pm 6.1$ & $319.2 \pm 6.1$ & $402.2 \pm 8.1$ \\
\hline a & 164.9 & 165.8 & 306.8 & 344.2 & 443.6 \\
\hline b & 164.0 & 159.2 & 292,8 & 319.2 & 402.2 \\
\hline
\end{tabular}

${ }^{2}$ Present results without the core-polarization effect on the dipole operator.

${ }^{b}$ Present results with the core-polarization cutoff radius chosen to reproduce the experimental polarizabilities. 
TABLE III. Quadrupole polarizabilities $\alpha_{2}\left(a_{0}^{5}\right)$.

TABLE V. Values of $C_{6}, C_{8}$, and $C_{10}$ in a.u. for Li-Li.

\begin{tabular}{|c|c|c|c|c|c|c|c|c|c|c|}
\hline Source & $\mathrm{Li}$ & $\mathrm{Na}$ & $\mathbf{K}$ & $\mathrm{Rb}$ & $\mathrm{Cs}$ & Source & - & $10^{-3} C_{6}$ & $10^{-5} C_{8}$ & $10^{-7} C_{10}$ \\
\hline$[15]$ & 1428 & & & & & [13] & & 1.391 & & \\
\hline$[18]$ & $1383^{--}$ & 1799 & 4587 & 5979 . & 9742 & [18] & & 1.389 & 0.8089 & 0.6901 \\
\hline [22] & 1486 & & & & & [21] & & 1.386 & & \\
\hline [23] & 1428 & & $\cdots--$ & & & {$[22]$} & & 1.445 & 0.8847 & 0.7902 \\
\hline [24] & 1423 & & & & & [5] & & 1.389 & & \\
\hline Present & 1424 & 1878 & 5000. & 6495 & 10462 & [39] & & 1.383 & 0.7578 & 0.4817 \\
\hline with & EII & 1 & & & & $\begin{array}{c}{[38]} \\
\text { Present }\end{array}$ & & $\begin{array}{l}1.360 \\
1.388 \\
\end{array}$ & 0.8324 & 0.7365 \\
\hline
\end{tabular}

$\mathrm{Li}$, but amounts to $10 \%$ for Cs. The increasing magnitude of the correction as the core size grows has been noted previously $[20,21]$. The experimental value of 164. $0 \pm 3.4$ [34] for $\mathrm{Li}$ has been reproduced by accurate many-electron calculations. The theoretical value of $164.1 a_{0}^{3}$ of Pipin and Bishop [24] is probably the most precise result. We include in Table II the values of Maeder and Kutzelnigg [18] and the calculations of Muller, Flesch, and Mayer [21] and Kello, Sadlej, and Faegri [35]. The close agreement suggests that the empirical correction procedure we have adopted adequately reflects the contribution from the core-electron transitions and should lead to reliable values of the van der Waals coefficients.

The quadrupole and octupole polarizabilities are given in Tables III and IV. For these the core corrections are small and may be neglected. There are no experimental data. For $\mathrm{Li}$ the most accurate value of the quadrupole polarizability is due to Pipin and Bishop [24] who, using correlated products of Hartree-type basis functions, obtained a value of $1423 a_{0}^{5}$ in perfect agreement with our value of $1424 a_{0}^{5}$. For the heavier alkali-metal atoms, the only useful comparison data are the results of Maeder and Kutzelnigg [18]. The values reported by Adelman and Szabo [36] are based on the method of Dalgarno and Pengelly [37], which is less accurate than the present procedure. Our values are considerably larger than those of Maeder and Kutzelnigg [18]. For Li, their value is certainly too small and we suspect it is a characteristic of their method.

A similar behavior occurs for octupole polarizabilities given in Table IV. Our values are again consistently larger than those of Maeder and Kulzelnigg [18]. For Li, there are useful comparison values of $\alpha_{3}$ from Knowles and Meath [22], who used an $R$-dependent $a b$ initio nonexpanded method for the calculation of dispersion energies. We have quoted the values from the basis-III column of their Table III. Their procedure overestimates $\alpha_{2}$ and may also do so for $\alpha_{3}$.

The coefficients $C_{6}, C_{8}$, and $C_{10}$ of the $R^{-6}, R^{-8}$, and $R^{-10}$ terms in the long-range interaction between two $\mathrm{Li}$ atoms are presented in Table $\mathrm{V}$, which also includes a

TABLE IV. Octupole polarizabilities $\alpha_{3}\left(a_{0}^{7}\right)$.

\begin{tabular}{cccccc}
\hline Source & $\mathrm{Li}$ & $\mathrm{Na}$ & $\mathrm{K}$ & $\mathrm{Rb}$ & $\mathrm{Cs}$ \\
\hline$[18]$ & 36495 & 51167 & 150161 & 212657 & 339920 \\
{$[22]$} & 41675 & & & & \\
Present & 39688 & 55518 & 176940 & 236850 & 395343 \\
\hline \hline
\end{tabular}

selection of previous results. The agreement amongst the different values of $C_{6}$ is close except for value of Knowles and Meath [22], which is too large. Our value of 1388 a.u. lies very near to the value of 1391 a.u. obtained in the most refined $a b$ initio many-electron calculation [13]. The self-consistent-field polarization potential method of [21] gives a value of 1386 a.u. For $C_{8}$ and $C_{10}$, our values lie between those of [22] which are likely to be overestimates and those of [18] and [38], which are likely to be underestimates, as suggested for [18] and [22] by the discussion of polarizabilities and for [38] by the low value of $C_{6}$. The large discrepancies with the results of Bussery and Aubert-Frecon [39] are unexpected.

Table VI is a compilation of values of $C_{6}, C_{8}$, and $C_{10}$ for the homonuclear cases $\mathrm{Na}-\mathrm{Na}, \mathrm{K}-\mathrm{K}, \mathrm{Rb}-\mathrm{Rb}$, and $\mathrm{Cs}-$ Cs. Our results for $C_{6}$ are consistently lower than those in [18] and are consistently larger for $C_{8}$ and $C_{10}$. Part of the difference can be attributed to the inclusion of the core-polarization potential and its contraction effect on the electric dipole operator. We have no explanation, however, for the occasional large differences with the results of Bussery and Aubert-Frecon [39]. With one exception, our results are consistent with the upper and lower bounds of Tang, Norbeck, and Certain [40] which however do not place rigorous bounds on the values.

None of the calculated values takes explicit account of contributions from core excitations. An uncertain cancellation of errors occurs in attempts to include core contributions [5], and it is probably more accurate to ignore

TABLE VI. Values of $C_{6}, C_{8}$, and $C_{10}$ in a.u. for alkali-metal atom pairs.

\begin{tabular}{rccccr}
\hline \hline & Source & Na-Na & K-K & Rb-Rb & Cs-Cs \\
\hline $10^{-3} C_{6}$ & {$[18]$} & 1.540 & 3.945 & 4.768 & 6.855 \\
& {$[21]$} & 1.518 & 3.574 & & \\
& {$[5]$} & 1.470 & 3.680 & 4.350 & 6.660 \\
& {$[39]$} & 1.698 & 4.721 & 5.726 & 9.469 \\
& {$[38]$} & 1.330 & 3.780 & 4.700 & 6.500 \\
& Present & 1.472 & 3.813 & 4.426 & 6.331 \\
& & & & & \\
$10^{-5} C_{8}$ & {$[18]$} & 1.098 & 3.834 & 5.244 & 9.025 \\
& {$[39]$} & 1.028 & 3.894 & 6.115 & 12.910 \\
& Present & 1.119 & 4.096 & 5.506 & 9.630 \\
$10^{-7} C_{10}$ & {$[18]$} & 1.036 & 4.522 & 6.836 & 13.010 \\
& {$[39]$} & 0.6939 & 4.069 & 6.316 & 14.510 \\
& Present & 1.107 & 5.248 & 7.665 & 15.200 \\
\hline \hline
\end{tabular}


TABLE VII. Dispersion coefficients in a.u. for different alkali-metal-atom interactions.

\begin{tabular}{|c|c|c|c|}
\hline & $C_{6}$ (units of $10^{3}$ ) & $C_{8}$ (units of $10^{5}$ ) & $C_{10}$ (units of $10^{7}$ ) \\
\hline $\mathrm{Li}-\mathrm{Na}$ & $1.460,^{\mathrm{a}} 1.427,1.448^{\mathrm{b}}$ & 1.068 & 0.982 \\
\hline Li-K & $2.334,^{a} 2.293,2.219^{b}$ & 2.517 & 2.651 \\
\hline Li-Rb & 2.469 & 3.137 & 3.413 \\
\hline $\mathrm{Li}-\mathrm{Cs}$ & 2.934 & 4.586 & 5.303 \\
\hline $\mathrm{Na}-\mathrm{K}$ & $2.443,^{\mathrm{a}} 2.348,2.309^{\mathrm{b}}$ & 2.614 & 2.949 \\
\hline $\mathrm{Na}-\mathrm{Rb}$ & 2.526 & 3.250 & 3.784 \\
\hline $\mathrm{Na}-\mathrm{Cs}$ & 2.993 & 4.727 & 5.844 \\
\hline $\mathbf{K} \cdot \mathbf{R} \mathbf{b}$ & 4.108 & 5.123 & 6.726 \\
\hline $\mathrm{K}-\mathrm{Cs}$ & 4.903 & 7.547 & 10.369 \\
\hline $\mathrm{Rb}-\mathrm{Cs}$ & 5.286 & 8.120 & 11.793 \\
\hline
\end{tabular}

"References [18].

${ }^{\mathrm{b}}$ Reference [21].

them [18], particularly since the core-polarization contribution to the dipole polarizability has been arranged so that the experimental values are reproduced. Probable upper limits to this error have been given by Dalgarno and Davision [5].

For the heteronuclear cases, we have listed the values for the dispersion coefficients in Table VII. The magnitudes of the dispersion coefficients lie between the values for the corresponding homonuclear cases. For instance, the $C_{6}$ coefficient for $\mathrm{Na}-\mathrm{K}$ falls between the value of $C_{6}$ for $\mathrm{Na}-\mathrm{Na}$ and the value of $C_{6}$ for $\mathrm{K}-\mathrm{K}$. Table VII also includes results from [18] and [21] for the $C_{6}$ coefficients. Once again, the values in [18] are larger than ours, mainly due to their neglect of the core-polarization effects.

It is often assumed that $C_{10}=1.3\left(C_{8}^{2} / C_{6}\right)$ [41]. For the cases in Tables V-VII, the ratio of $C_{10}$ to $\left(C_{8}^{2} / C_{6}\right)$ varies between 0.74 and 1.48 .

\section{CONCLUSIONS}

Using an efficient numerical algorithm originally developed for obtaining absorption line profiles, we calculate the dispersion coefficients in the long-range $1 / R$ expansion of the electrostatic interaction between two ground-state alkali-metal atoms. First, we describe the motion of the valence electron in the presence of the closed-shell core by the well-established method of parametric model potential. This angular-momentumdependent central potential includes the effect of the core polarizability which is cut off at the core boundary to eliminate the unphysical nature of the polarization potential near the nucleus. We then evaluate the dispersion energy coefficients by integrating over the dynamic electric multipole polarizabilities of the product dimer at imaginary frequencies. The polarizabilities are obtained by solving two coupled inhomogeneous differential equations. We have extended the single-channel Numerov differential equation solver method to handle coupled inhomogeneous equations. In this vein, our calculations in the spirit of perturbation theory are "exact", i.e., no approximation is made in solving the inhomogeneous equations.

We also investigate the effect of the core polarization on the electric dipole of the valence electron and show that this effect leads to a general lowering of the $1 / R^{6}$ dispersion energies, as expected in view of the fact that the polarization of the core amounts to a contraction of the dipole moment. We present a comprehensive compilation of the multipole polarizabilities and dispersion energies for alkali-metal dimers.

\section{ACKNOWLEDGMENTS}

H.R.S. and A.D. were supported by the U.S. Department of Energy, Division of Chemical Sciences, Office of Basic Energy Sciences, Office of Energy Research. M.M. was supported by the Smithsonian Institution.
[1] C. R. Monroe, E. L. Cornell, C. A. Sackett, C. J. Myatt, and C. E. Wieman, Phys. Rev. Lett. 70, 414 (1993).

[2] M. Prentiss, A. Cable, J. E. Bjorkholm, S. Chu, E. L. Raab, and D. E. Pritchard, Opt. Lett. 13, 452 (1988).

[3] P. S. Julienne, A. M. Smith, and K. Burnett, Adv. At. Mol. Opt. Phys. 30, 141 (1992).

[4] A. Dalgarno, Adv. Phys. 11, 281 (1962).

[5] A. Dalgarno and W. D. Davision, Adv. At. Mol. Phys. 2, 1 (1966); A. Dalgarno, Adv. Chem. Phys. 12, 143 (1967).

[6] P. R. Fontana, Phys. Rev. 123, 1865 (1961).

[7] T. Y. Chang, Rev. Mod. Phys. 39, 911 (1967).

[8] P. W. Langhoff and M. Karplus, J. Chem. Phys. 53, 233 (1970).
[9] H. Pauly and J. P. Toennies, Adv. At. Mol. Phys. 1, 195 (1965).

[10] C. Mavroyannis and M. J. Stephen, Mol. Phys. 5, 629 (1962).

[11] A. D. McLachlan, Proc. R. Soc. London, Ser. A 271, 387 (1962).

[12] Y. M. Chan and A. Dalgarno, Proc. Phys. Soc. London, 85, 1455 (1965).

[13] G. M. Stacey and A. Dalgarno, J. Chem. Phys. 48, 2515 (1968).

[14] H. J. Werner and W. Meyer, Phys. Rev. A 13, 13 (1976).

[15] E.-A. Reinsch and W. Meyer, Phys. Rev. A 14, 915 (1976).

[16] G. D. Zeiss, W. J. Meath, J. C. F. MacDonald, and D. J. 
Dawson, Can. J. Phys. 55, 2080 (1977)

[17] J. E. Kouba and W. J. Meath, Mol. Phys. 34, 1351 (1977).

[18] F. Maeder and W. Kutzelnigg, Chem. Phys. 42, 195 (1979).

[19] P. A. Christiansen and K. S. Pitzer, Chem. Phys. Lett. 22, 434 (1982).

[20] P. Fuentealba, J. Phys. B 15, L555 (1982).

[21] W. Muller, J. Flesch, and W. Meyer, J. Chem. Phys. 80, 3297 (1984).

[22] P. J. Knowles and W. J. Meath, Chem. Phys. Lett. 124, 164 (1986).

[23] G. Maroulis and A. Thakkar, J. Phys. B 22, 2439 (1989).

[24] J. Pipin and D. M. Bishop, Phys. Rev. A 45, 2736 (1992).

[25] C. H. Greene, Phys. Rev. A 42, 1405 (1990).

[26] H. R. Sadeghpour and A. Dalgarno, J. Phys. B 25, 4801 (1992).

[27] A. Dalgarno and J. T. Lewis, Proc. R. Soc. London, Ser. A 233, 70 (1955).

[28] J. M. Blatt, J. Comput. Phys. 1, 382 (1967).

[29] Y. M. Chan and A. Dalgarno, Mol. Phys. 9, 349 (1965).

[30] C. Laughlin and G. A. Victor, Adv. At. Mol. Phys. 25, 163 (1988), and references therein.

[31] C. H. Greene and M. Aymar, Phys. Rev. A 44, 1773
(1991), and references therein.

[32] R. P. Wood, C. H. Greene, and D. Armstrong, Phys. Rev. A 47, 1981 (1993).

[33] C. Bottcher and A. Dalgarno, Proc. R. Soc. London Ser. A 340, 187 (1974); S. Hameed, A. Herzenberg, and M. G. James, J. Phys. B 1, 822 (1968).

[34] R. W. Molof, H. J. Schwartz, T. M. Liller, and B. Bederson, Pnys. Rev. A 10, 1131 (1974).

[35] V. Kello, A. J. Sadlej, and K. Faegri, Phys. Rev. A 47, 1715 (1993).

[36] S. A. Adelman and A. Szabo, J. Chem. Phys. 58, 687 (1873).

[37] A. Dalgarno and R. M. Pengelly, Proc. Phys. Soc. London 89, 503 (1966).

[38] M. L. Manakov and V. O. Ovsiannikov, J. Phys. B 10, 569 (1985).

[39] B. Bussery and M. Aubert-Frecon, J. Chem. Phys. 82, 3224 (1985).

[40] K. T. Tang, J. M. Norbeck, and P. R. Certain, J. Chem. Phys. 64, 3063 (1976).

[41] W. T. Zemke and W. C. Stwalley, J. Phys. Chem. 97, 2053 (1993). 